\title{
The Ly-edge paradox and the need for obscured QSOs
}

\author{
R. Maiolino ${ }^{1}$, M. Salvati ${ }^{1}$, A. Marconi ${ }^{1}$, and R. R. J. Antonucci ${ }^{2}$ \\ 1 Osservatorio Astrofisico di Arcetri, Largo E. Fermi 5, 50125 Firenze, Italy \\ 2 Physics Department, University of California, Santa Barbara, CA 93106, USA
}

Received 16 March 2001 / Accepted 1 June 2001

\begin{abstract}
Based on the most recent QSO ultraviolet spectra, the covering factor of the clouds of the Broad Line Region (BLR) is about 30\%, or larger. This value would imply that in at least $30 \%$ of the QSOs our line of sight crosses one, or more, BLR clouds and, in the latter case, the UV spectrum should show a sharp Ly-edge in absorption. This Ly-edge in absorption is never observed. This paradox is solved if, as suggested by various authors, the BLR is flattened and the dusty gas in the outer parts, on the same plane, prevents the observation along the lines of sight passing through the BLR clouds. The objects observed edge-on (with respect to the flattened BLR) would be classified as obscured QSOs or, within the framework of the unified model, type 2 QSOs. The covering factor of the BLR constrains the fraction of obscured QSOs to be QSO2/QSO1 > 0.5. This lower limit is already high with respect to the number of candidate type 2 QSOs claimed so far. We discuss this constraint in relation to recent AGN surveys.
\end{abstract}

Key words. quasars: general - galaxies: nuclei - ultraviolet: galaxies

\section{Introduction}

The existence of obscured QSOs, or type 2 QSOs, has been a hotly debated issue since the formulation of the first unified theories. While the relation between Seyfert $1 \mathrm{~s}$ and their obscured counterparts, the Seyfert $2 \mathrm{~s}$, has been thoroughly assessed, at higher luminosities $\left(L_{\mathrm{bol}}>\right.$ $10^{45} \mathrm{erg} \mathrm{s}^{-1}$ ) we are still at the stage of questioning whether a significant population of the obscured counterparts of QSOs exist or not. Only for the sub-class of radio-loud QSOs a population of obscured counterparts was identified (the narrow line radio galaxies). Yet, radioloud AGNs are only a minor fraction of the whole AGN population. Among radio-quiet AGNs there are only a few cases of candidate type 2 QSOs. Most of the ultraluminous infrared galaxies (ULIRGs, which are among the best candidates to host hidden QSOs) appear mostly powered by starburst activity, while evidences for a hidden AGN is only found on the high luminosity tail of this population (Lutz et al. 1998; Evans et al. 1998; Murphy et al. 2001; Veilleux et al. 1999). Also, even in those ULIRGs showing evidence for a hidden AGNs, its contribution to the bolometric luminosity is not well constrained, except for a few cases (e.g. Franceschini et al. 2000). For what concerns surveys in the hard X-rays, which should be less affected by obscuration than optical and soft X-ray surveys, only

Send offprint requests to: $\mathrm{R}$. Maiolino, e-mail: maiolino@arcetri.astro.it two cases of type 2 QSO have been identified (Norman et al. 2001; Crawford et al. 2001).

While the evidence for a "classical" population of type 2 QSOs is marginal, a population of broad-line QSOs whose continuum is redder than classical color-selected AGNs was found in radio and X-ray surveys (Webster et al. 1995; Kim \& Elvis 1999; Fiore et al. 1999; Risaliti et al. 2001; Maiolino et al. 2000). Although in some of these objects the red continuum is ascribed to the contribution from the host galaxy or to synchrotron emission (especially for the radio-loud objects), there is evidence that in several of them dust reddening and extinction play a major role.

In this paper we demonstrate that evidence for the existence of a large population of optically obscured QSOs is inferred, ironically, by the UV spectra of unobscured QSOs.

\section{The covering factor of the BLR}

The covering factor of the BLR has been discussed by several authors in the past, based on the comparison between the hydrogen recombination lines and the slope of the optical-UV continuum extrapolated beyond the Ly-edge or by using models for the shape of the ionizing continuum. By using the Ly $\alpha$ as a photon counter it was found that the covering factor is between $5 \%$ and $10 \%$ (e.g. Oke \& Korycansky 1982; Carswell \& Ferland 1988). By using 
the $\mathrm{EW}$ of $\mathrm{H} \beta$ Binette et al. (1993) derived an even higher covering factor $(\sim 40 \%)$.

Recent UV spectra of QSOs have shown that the fraction of ionizing photons is significantly lower than estimated in the past. Zheng et al. (1997, hereafter Z97) have created a composite UV spectrum out of HST spectra of 101 QSOs with redshift between 0.33 and $\sim 1.5$ (i.e. without large effects from the intervening $\operatorname{Ly} \alpha$ forest absorption). One of the most interesting features of the composite spectrum is that the slope in the extreme UV region is significantly steeper than was thought in the past. More recently, Kriss (2000) generated a composite with 248 QSO spectra observed with HST; the result is in good agreement with Z97. As first noted by Laor et al. (1997) the fraction of ionizing photons is about a factor of four lower than in models adopted previously (when normalized to the near-UV/optical light). Since the equivalent width of the emission lines in Z97 is not very different from previous measurements, this implies that the covering factor must be higher by a factor of about four than estimated in the past. More specifically, by simply using the Ly $\alpha$ flux in the composite spectrum of Z97 as a ionizing photon counter, along with the number of ionizing photons actually derived from the same spectrum, a covering factor of $27 \%$ is inferred.

By using more complex models of the BLR the inferred covering factor does not change significantly. For instance, by using the LOC model by Korista et al. (1998), adapted to the spectral shape observed by Z97, and by simply comparing the expected EW of Ly $\alpha$ and HeII $1640 \AA$ A with the values measured by Z97, we obtain a covering factor of about $30 \%$.

Several authors have proposed that the ionizing radiation is not emitted isotropically by the accretion disk and that the edge-on lines of sight see a harder continuum (e.g. Netzer 1987; Sun \& Malkan 1989; Laor \& Netzer 1989). If, as proposed by Netzer (1987) and by Korista et al. (1997) and as discussed more in detail below, the disk is preferentially observed pole-on by us, while the BLR is flattened on the plane of the disk, then the anisotropic emission implies an even higher covering factor.

A larger covering factor is also obtained if the composite spectrum of Z97 is included in the calculations of Binette et al. (1993).

Summarizing, we will assume $30 \%$ as a lower limit to the covering factor of the BLR.

\section{The missing Ly-edge}

If the BLR clouds had a spherically symmetric distribution around the ionizing source then, given the relatively large covering factor of the BLR, we would expect that the line of sight towards the UV ionizing source intercepts a cloud in more than $30 \%$ of the cases, hence we would expect a sharp Lyman absorption edge in more than $30 \%$ of the QSOs or, equivalently, the composite spectrum should show a sharp Lyman absorption edge deeper than $30 \%$ of the continuum red-ward of the Ly-edge. This remains true

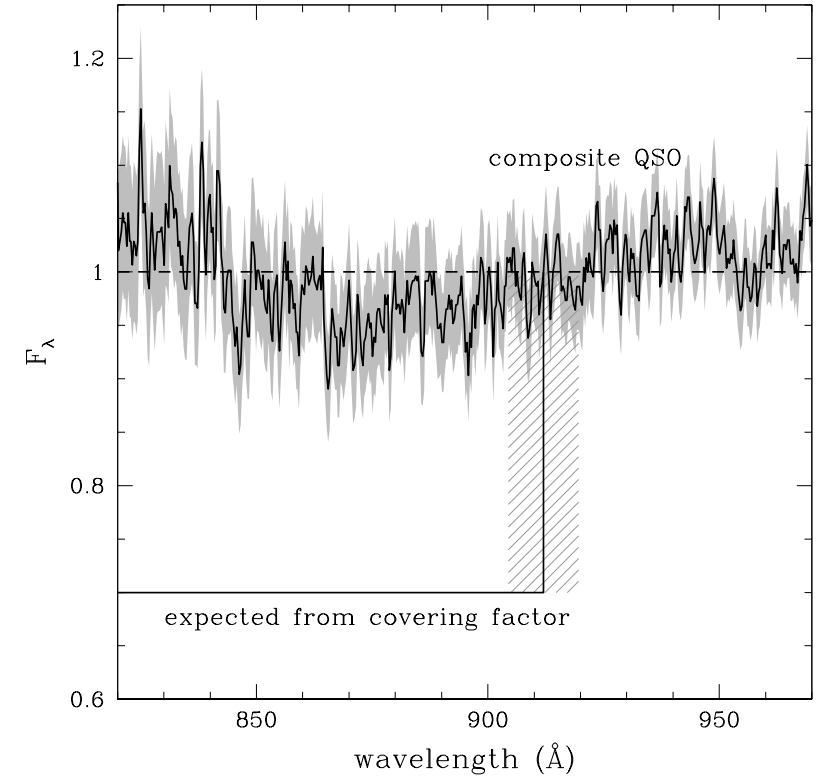

Fig. 1. Composite QSO spectrum derived by Z97, divided by the power-law fitting the continuum in the vicinity of the Lyedge (and normalized by the continuum at the Ly-edge). The shaded region gives the error on the mean. The solid broken line gives the expected depth of the Ly-edge by assuming a covering factor of $30 \%$ (which is the lower limit on the derived in Sect. 2). The hatched region gives the uncertainty due to the velocity spread of the BLR clouds.

even in the case that the ionizing UV source has dimensions comparable or larger than the clouds.

Figure 1 shows the composite QSO spectrum of Z97 (both radio-quiet and radio-loud QSOs) around the Lyedge divided by the power-law which has been fitted to the spectrum in the same region and normalized at the Lyedge. The shaded region indicates the error on the mean. The composite spectrum does not show any indication for the sharp absorption edge expected by absorbing gas in the BLR along our line of sight. The lower straight line in Fig. 1 shows the upper limit on the continuum level blueward of the Ly-edge expected from the covering factor of the BLR, which is clearly inconsistent with the composite QSO spectrum.

We can even state that, to our knowledge, no single QSO has shown evidence for the presence of a Ly absorption edge ascribed to intrinsic absorption from BLR clouds along the line of sight (Antonucci et al. 1989; Koratkar \& Blaes 1999). Some sharp Ly-edge absorption in a few QSOs was totally ascribed to external systems at lower redshift.

This is not the first time that the problem of the missing Ly-edge is highlighted (e.g. Antonucci et al. 1989). Yet, the larger covering factor of the BLR inferred by the recent data makes this issue even more serious.

One might argue that if the absorbing clouds are moving along our line of sight (outflowing and/or inflowing) then the resulting Ly-edge in the composite spectrum would be broadened and therefore more difficult to detect. However, given the velocity dispersion in the BLR, 
the broadening of the Ly-edge would be at most as high as the width of the emission lines and, therefore, an absorption edge as deep as $30 \%$ of the continuum should be detectable. The hatched vertical region in Fig. 1 shows the width of of the Ly-edge which would be produced by a velocity dispersion of $5000 \mathrm{~km} \mathrm{~s}^{-1}$.

The emission of a Ly continuum, expected at some level by BLR models (Korista \& Goad 2001; Davidson 1976), could partly fill up the Ly edge absorption. However, such a Ly continuum emission has never been observed in any object, not even in those showing a Balmer continuum. Also, while the photoelectric absorption should extend at least to the soft X-rays $\left(N_{\mathrm{H}}(\mathrm{BLR}) \approx\right.$ $10^{22}-10^{23} \mathrm{~cm}^{-2}$ ), the Ly continuum emission would rapidly decrease blue-ward of the Ly-edge and at $700 \AA$ would be less than $10 \%$ of the value at the Ly-edge. On the contrary, the Z97 spectrum does not show any indication of absorption down to $300 \AA$.

Binette et al. (1993, and references therein) proposed that the observed UV-optical continuum consists mostly of scattered/reprocessed radiation while the intrinsic spectrum is obscured along our line of sight in most AGNs (even in type 1). This would explain the lack of Ly-edge absorption. However, the intrinsic spectrum should be observable in the hard X-rays with a prominent photoelectric cut-off, while most of the optically selected QSOs (which roughly match the sample used by Z97) observed in the X-rays do not show any absorption cutoff, with only a few exceptions (Reeves \& Turner 2000; Maiolino et al. 2001).

Dust within the BLR clouds would possibly explain the lack of the Ly-edge absorption, since it would absorb the UV continuum also red-ward of the Ly-edge. However, the BLR is located inside the sublimation radius of dust and even the shielding the ionizing radiation due to the gas is probably unable to allow the survival of grains in the dark side of the clouds.

\section{The paradox and the need for obscured QSOs}

The paradoxical result of this analysis is that although the BLR clouds cover a large fraction of the sky to the nuclear source, whenever we observe a QSO our line of sight never intercepts these clouds.

The origin of this paradox is probably related to the assumption that the BLR has a spherically symmetric distribution around the ionizing source. If the BLR clouds are mostly distributed in a flattened geometry and the observer's lines of sight are mostly pole-on, then the BLR emission is observed, but no Ly-edge in absorption is detected. Then the edge-on lines of sight, which should intercept the ionized clouds and show the Ly-edge absorption, are never observed because of the dusty gas on the plane of the BLR, but outside the sublimation radius, and which is responsible for absorbing and reddening the QSOs observed "edge-on", therefore preventing their detection in color-selected surveys.

Within the framework of the unified model the outer dusty gas would be identified with the "obscuring torus" and the QSOs observed edge-on would be identified as type 2, obscured QSOs. The fact that no QSO with Ly absorption edge has ever been observed implies that not only the dusty torus must be coplanar with the flattened broad line region, but also that the covering factor of the torus must be larger than the covering factor of the broad line region. Within this scenario a lower limit to the fraction of obscured QSOs is given by the covering factor of the BLR. As a consequence, obscured (type 2) QSOs must at least be as numerous as half of the unobscured population of QSOs.

A flattened distribution for the BLR was proposed by various authors in the past, both based on theoretical and on observational arguments (Netzer 1987; Collin-Souffrin \& Dumont 1990; Wills \& Brotherton 1995; Wanders et al. 1995; Goad \& Wanders 1996). We note that the scenario of a flattened BLR coplanar with the obscuring torus is consistent with the finding that the hard X-ray spectra of some Seyfert 2 galaxies show evidence for a double absorber (see review in Maiolino 2001). While the (total) absorber with lower $N_{\mathrm{H}}$ is ascribed to the obscuring torus, the absorber with higher $N_{\mathrm{H}}$ (typically a few times $10^{23} \mathrm{~cm}^{-2}$ ) has a partial covering (generally $>30 \%$ ) and it is ascribed to BLR clouds along the line of sight.

\section{Enough obscured QSOs?}

The problem of the shortage of obscured QSOs is not new and has been widely discussed in the past. However, with the exception of the requirement of X-ray absorbed QSOs to account for the X-ray number counts by Gilli et al. (2001), there were no other observational or theoretical constraints which required the existence of large population of (optically) obscured QSOs. In this paper we link the problem of the missing Ly-edge with the issue of the shortage of obscured QSOs and constrain the minimum fraction of obscured QSOs. In this section we compare this lower limit with the fraction of obscured QSO known so far.

The lower limit on the fraction of type 2 QSOs derived in the former section (QSO2/QSO1 > 0.5) is already quite high when compared with the handful of (candidate) type 2 QSOs discovered so far. While in the case of radio loud AGNs the fraction of narrow line radio galaxies is enough to account for the Ly-edge paradox (NLRG/RLQSO 1, Singal 1993), for what concerns radio-quiet AGNs, as mentioned in the introduction, there are only a few bona fide type 2 QSOs discovered so far.

It should be noted that the samples of QSOs used to study their extreme UV spectrum are generally biased in favor of UV bright objects and, therefore, heavily biased against the so-called red QSOs, which were discussed in the Introduction. If the properties of most red QSOs are ascribed to dust extinction, then they could be the population of QSOs observed edge-on which should show Ly-edge in absorption due to BLR clouds, i.e. the population of "obscured" QSOs. Is the number of red QSO large enough to account for the missing Ly-edge? 
The fraction of red QSOs in soft X-ray selected and radio selected samples is below $20 \%$ of the whole broad line QSO population (Kim \& Elvis 1999 and references therein), i.e. lower than the minimum fraction of obscured QSOs inferred from the BLR covering factor $(>30 \%)$. However, recently Risaliti et al. (2001) have found indications, among grism-selected QSOs, for a larger fraction of red, absorbed objects $(\sim 50 \%)$.

Hard X-rays are probably the most suited for the search of obscured QSOs. The identification of the optical counterparts of the recent deep and medium-deep Chandra surveys has revealed some powerful (type 1) QSOs (Barger et al. 2001; Giacconi et al. 2001; Fiore et al. 2000; Hornschemeier et al. 2000; Tozzi et al. 2001; Mushotzky et al. 2000; Brandt et al. 2000, 2001; Crawford et al. 2001). Several narrow line AGNs have been identified with luminosities in the Seyfert range. Some obscured AGNs with 2-10 keV luminosity of a few times $10^{44} \mathrm{erg} / \mathrm{s}$ have been referred to as "obscured QSOs", but we consider these objects just as bright Seyfert 2s. For comparison, NGC 6240, whose de-absorbed 2-10 keV luminosity is $\sim 2 \times 10^{44} \mathrm{erg} / \mathrm{s}\left(H_{0}=70\right.$, Vignati et al. 1999), has an infrared total luminosity ${ }^{1}$ of only $7 \times 10^{11} L_{\odot}$ which does not even classify it as a ULIRG and which is only a factor of two higher than the total IR luminosity of the local Seyfert 2 archetype NGC 1068. Actually, to our knowledge the Chandra surveys have so far identified only two type 2 QSOs with luminosities of $L_{2-10 \mathrm{keV}} \approx 10^{45} \mathrm{erg} \mathrm{s}^{-1}$ (Norman et al. 2001; Crawford et al. 2001), although for one of them the estimation of the intrinsic luminosity is subject to large uncertainty. Possibly, most of the QSO2s have still to be identified among the fainter counterparts of the Chandra sources, but this seems unlikely since several Sy2s have already been identified (even at high redshift). Yet, it should be noted that several of the (type 1) QSOs identified in the Chandra surveys have a continuum which is redder than color selected QSOs, therefore suggesting that these Chandra QSOs are affected by some dust reddening. Some indication of gas obscuration is also inferred from their X-ray spectral slope which, for some of them, is slightly harder than in classical QSOs. The fraction of these mildly obscured QSOs (either red or with harder X-ray spectra) is certainly enough to account for the Lyedge paradox.

The limited sky coverage of the current Chandra surveys is not optimal for a statistical analysis of the properties of QSOs since their density in the sky is very low. The hard X-ray surveys obtained by ASCA and BeppoSAX (Akiyama et al. 2000; Fiore et al. 1999; Fiore et al. 2001) are certainly shallower, but cover a much larger area of the sky. However, even in these surveys, despite the large fraction of (type 1) QSOs identified, no clear cases for type 2 QSOs were found. Yet, a significant fraction of mildly obscured QSOs was found: red QSOs or (type 1) QSOs with indication of X-ray absorption.

\footnotetext{
${ }^{1} L_{\mathrm{ir}}=L(8-1000 \mu \mathrm{m})\left(\sim L_{\mathrm{bol}}\right)$ as defined in Sanders \& Mirabel (1996).
}

Summarizing, although only two QSO2s have been identified in the hard X-ray surveys so far, a fraction of (mildly) obscured QSOs has been identified which would be consistent with the lower limit on the obscured-tounobscured ratio inferred by the Ly-edge paradox.

Another possibility is that most of the type 2 QSOs are Compton thick and, therefore, have been missed even by the hard X-ray surveys because they are too dim in the $2-10 \mathrm{keV}$ band. However, this scenario seems unlikely at least for the most recent surveys, given that one of the type 2 QSOs discovered in the Chandra fields is actually a Compton thick object at high redshift (Norman et al. 2001), therefore highlighting the capability of Chandra to detect even Compton thick type 2 QSOs. Alternatively, these Compton thick type 2 QSOs have already been detected, but misidentified as Seyfert 2s (or simply Narrow Line AGNs with $\left.L_{\text {hard-X }}<10^{44} \mathrm{erg} \mathrm{s}^{-1}\right)$. Indeed, in the Compton thick case the intrinsic hard X-ray luminosity would probably be $2-3$ orders of magnitude higher than inferred by the (reflection-dominated) observed flux. However, the ratio between the far-IR and the X-ray cosmic background constrains most of the absorbed AGNs not to be "reflection dominated". More specifically it can be shown that a fraction of Compton thick, reflection-dominated AGNs contributing more than $\sim 10 \%$ of the $2-10 \mathrm{keV}$ background would over-produce the COBE background at $\sim 140 \mu \mathrm{m}$ (see also Risaliti et al. in preparation).

\section{Conclusions}

The UV spectra of QSOs indicate that the covering factor of the clouds of the Broad Line Region must be larger than $\sim 30 \%$. This covering factor would imply that more than $30 \%$ of the lines of sight should intersect a BLR cloud and show a sharp Ly-edge in absorption. This intrinsic absorption edge has never been observed in single QSOs spectra nor in a composite spectrum of 100-200 QSOs. This paradoxical result is in keeping with previous suggestions that the BLR is not distributed isotropically around the nuclear source but flattened; within this scenario the lines of sight passing through the BLR clouds are not observed in the UV because of dusty gas associated with the outer parts of the BLR which obscures the whole nuclear region. The QSOs observed along the latter lines of sight would be classified as red QSOs or, if the absorption is large enough, as type 2 QSOs. Within this scenario the covering factor of the BLR provides a lower limit to the fraction of obscured QSOs and, more specifically, QSO2/QSO1 > 0.5. This lower limit is already much higher than the fraction observationally obtained so far.

A possibility is that "pure" type 2 QSOs are really sparse and the population of obscured QSOs inferred from the Ly-edge paradox is accounted for by the mildly obscured QSOs (the red QSOs and those type 1 QSOs with some X-ray absorption) found in hard X-ray surveys. A minor fraction of type 2 QSOs might be Compton thick; 
these have either been lost even by hard X-ray surveys, or have been misclassified as Seyfert $2 \mathrm{~s}$.

If mildly obscured QSOs found in the hard X-ray surveys (which have $N_{\mathrm{H}}<10^{22}-10^{23} \mathrm{~cm}^{-2}$ ) account for the obscured QSOs requested by the missing Ly-edge, when compared with the $N_{\mathrm{H}}$ distribution of Sy2s (most of which have $N_{\mathrm{H}}>10^{23} \mathrm{~cm}^{-2}$, Risaliti et al. 1999), this implies that the average $N_{\mathrm{H}}$ decreases with the luminosity. This finding is supported by similar results on the radio-loud QSOs (Singal 1993; Lawrence 1991; Woltjer 2001).

Finally, our results would imply that most of the hard $\mathrm{X}$-ray background is produced by heavily obscured Seyfert $2 \mathrm{~s}$ (as the ongoing Chandra identification campaigns seem to confirm) and that mildly obscured QSOs only account for the $2-10 \mathrm{keV}$ number counts at intermediate fluxes $\left(\sim 10^{-13} \mathrm{erg} \mathrm{s}^{-1} \mathrm{~cm}^{-2}\right.$, Gilli et al. 2001).

Acknowledgements. We are grateful to Kirk Korista and Lo Woltjer for useful discussions and to the anonymous referee for useful comments. We thank Wei Zheng for providing the electronic version of the composite QSO spectrum. RM is grateful to Emilia Caporale for "suggesting" the title of this paper. This work was partially supported by the Italian Ministry for University and Research (MURST) under grant Cofin00-02-36 and by the Italian Space Agency (ASI) under grant 1/R/27/00.

\section{References}

Akiyama, M., Ohta, K., Yamada, T., et al. 2000, ApJ, 532, 700 Antonucci, R. R. J., Kinney, A. L., \& Ford, H. C. 1989, ApJ, 342,64

Barger, A. J., Cowie, L. L., Mushotzky, R. F., \& Richards, E. A. 2001, AJ, 121, 662

Binette, L., Fosbury, R. A., \& Parker, D. 1993, PASP, 105, 1150

Brandt, W. N., Hornschemeier, A. E., Schneider, D. P., et al. 2000, AJ, 119, 2349

Brandt, W. N., Hornschemeier, A. E., Alexander, D. M., et al. 2001, AJ, in press [astro-ph/0102411]

Carswell, R. F., \& Ferland, G. J. 1988, MNRAS, 235, 1121

Collin-Souffrin, S., \& Dumont, A. M. 1990, A\&A, 229, 292

Crawford, C. S., Ghandi, P., Fabian, A. C., et al. 2001, MNRAS, submitted [astro-ph/0106067]

Davidson, K. 1976, ApJ, 207, 710

Evans, A. S., Sanders, D. B., Cutri, R. M., et al. 1998, ApJ, 506,205

Fiore, F., La Franca, F., Giommi, P., et al. 1999, MNRAS, 306, L55

Fiore, F., La Franca, F., Vignali, C., et al. 2000, NewA, 5, 143

Fiore, F., et al. 2001, in X-ray Astronomy '999: Stellar Endpoints, AGNs and the Diffuse X-ray Background, ed. G. Malaguti, G. G. C. Palumbo, \& N. White, Astroph. Lett. Comm, in press [astro-ph/0007473]

Franceschini, A., Bassani, L., Cappi, M., et al. 2000, A\&A, 200,58
Giacconi, R., Rosati, P., Tozzi, P., et al. 2001, ApJ, 551, 624

Gilli, R., Salvati, M., \& Hasinger, G. 2001, A\&A, 366, 407

Goad, M. R., \& Wanders, I. 1996, MNRAS, 255, 663

Hornschemeier, A. E., Brandt, W. N., Garmire, G. P., et al. 2000, ApJ, 541, 49

Kim, D. -W., \& Elvis, M. 1999, ApJ, 516, 9

Koratkar, A., \& Blaes, O. 1999, PASP, 111, 1

Korista, K., Ferland, G., \& Baldwin, J. 1997, ApJ, 487, 555

Korista, K., Baldwin, J., \& Ferland, G. 1998, ApJ, 507, 24

Korista, K. T., \& Goad, M. 2001, ApJ, in press [astro-ph/0101117]

Kriss, G. A. 2000, in Probing the Physics of AGN by Multiwavelength Monitoring, GSFC workshop, in press [astro-ph/0008492]

Hamann, F., \& Ferland, G. 1999, ARA\&A, 37, 487

Laor, A. 1997, ApJ, 477, 93

Laor, A., \& Netzer, H. 1989, MNRAS, 238, 897

Lawrence, A. 1991, MNRAS, 252, 586

Lutz, D., Spoon, H. W. W., Rigopoulou, D., Moorwood, A. F. M., \& Genzel, R. 1998, ApJ, 505, L103

Maiolino, R., Salvati, M., Antonelli, L. A., et al. 2000, A\&A, 355, L47

Maiolino, R. 2001, in X-ray Astronomy '999: Stellar Endpoints, AGNs and the Diffuse X-ray Background, ed. G. Malaguti, G. G. C. Palumbo, \& N. White, Astroph. Lett. Comm, in press [astro-ph/0007473]

Maiolino, R., Marconi, A., Salvati, M., et al. 2001, A\&A, 365, 28

Murphy, T. W., Soifer, B. T., Matthews, K., Armus, L., Kiger, J. R. 2001, AJ, 121, 97

Mushotzky, R. F., Cowie, L. L., Barger, A. J., \& Arnauld, K. A. 2000, Nature, 404, 459

Netzer, H. 1987, MNRAS, 225, 55

Norman, C., Hasinger, G., Giacconi, R., et al. 2001, ApJ, in press [astro-ph/0103198]

Oke, J. B., Korycansky, D. G. 1982, ApJ, 255, 11

Reeves, J. N., \& Turner, M. J. L. 2000, MNRAS, 316, 234

Risaliti, G., Maiolino, R., \& Salvati, M. 1999, ApJ, 522, 157

Risaliti, G., Marconi, A., Maiolino, R., Salvati, M., \& Severgnini, P. 2001, A\&A, 371, 37

Sanders, D. B., Mirabel, I. F. 1996, ARA\&A, 34, 749

Singal, A. K. 1993, MNRAS, 262, L27

Sun, H. -H., \& Malkan, M. A. 1989, ApJ, 346, 68

Tozzi, P., Rosati, P., Nonino, M., et al. 2001, ApJ, in press [astro-ph/0103014]

Veilleux, S., Sanders, D. B., Kim, D.-C. 1999, ApJ, 522, 139

Vignati, P., Molendi, S., Matt, G., et al. 1999, A\&A, 349, L57

Wanders, I., Goad, M. R., Korista K. T., et al. 1995, ApJ, 453, L87

Webster, R. L., Francis, P. J., Peterson, B. A., Drinkwater, M. J., \& Masci, F. J. 1995, Nature, 375, 469

Wills, B. J., \& Brotherton, M. S. 1995, ApJ, 448, L81

Woltjer, L. 2001, in Quasars, AGNs and Related Research Across 2000, ESO proceed., in press

Zheng, W., Kriss, G. A., Telfer, R. C., Grimes, J. P., \& Davidsen, A. F. 1997, ApJ, 475, 469 (Z97) 